\title{
On electroweak symmetry breaking in the littlest Higgs model
}

\author{
A. Dobado ${ }^{1}$, L. Tabares ${ }^{1}$, S. Peñaranda ${ }^{2,3, a}$ \\ ${ }^{1}$ Departamento de Física Teórica I, Universidad Complutense de Madrid, 28040 Madrid, Spain \\ 2 CERN TH Division, Department of Physics, 1211 Geneva 23, Switzerland \\ 3 IFIC - Instituto de Física Corpuscular, CSIC - Universitat de València, 46071 Valencia, Spain
}

Received: 17 August 2006 / Revised version: 9 November 2006 /

Published online: 20 February 2007 - (C) Springer-Verlag / Società Italiana di Fisica 2007

\begin{abstract}
In $\mathrm{SU}(5) / \mathrm{SO}(5)$ little Higgs models radiative corrections give rise to $\mathrm{SU}(2)_{\mathrm{L}} \times \mathrm{U}(1)_{Y}$ symmetry breaking. In this work we start a program for a detailed determination of the relevant terms of the effective Higgs potential by computing the contribution of the $t, b$ and $T$ quarks at the one-loop level, as a starting point for a higher-loop computation. In spite of the fact that some two-loop level contributions are well known to be important, we use our preliminary one-loop result to illustrate that, by demanding the effective potential to reproduce exactly the standard model Higgs potential, and in particular the relation $m_{H}^{2}=2 \lambda v^{2}=2 \mu^{2}$, it will be possible to set new constraints on the parameter space of the littlest Higgs model when the computation of all the relevant contributions to the effective Higgs potential is completed.
\end{abstract}

\section{Introduction}

In recent years a lot of work has been devoted to the socalled little Higgs models (see [1,2] for recent reviews). These models use an old suggestion by Georgi and Pais [3, 4], in which the Higgs boson is assumed to be a (pseudo-) Goldstone boson associated to some global spontaneous symmetry breaking [5-10]. For instance, in the case of the paradigmatic littlest Higgs (LH) model [11], SU(5) to $\mathrm{SO}(5)$ breaking is assumed to happen at some scale $f$. The Higgs field is just one of the corresponding 14 Goldstone bosons, and therefore it is, in principle, massless. The $\mathrm{SU}(5)$ subgroup $(\mathrm{SU}(2) \times \mathrm{U}(1))_{1} \times(\mathrm{SU}(2) \times \mathrm{U}(1))_{2}$ is gauged so that the axial $(\mathrm{SU}(2) \times \mathrm{U}(1))_{1-2}$ is spontaneously broken, with the gauge bosons typically being heavy $\left(W^{\prime a}\right.$ and $\left.B^{\prime}\right)$. The diagonal $(\mathrm{SU}(2) \times \mathrm{U}(1))_{1+2}=$ $\mathrm{SU}(2)_{\mathrm{L}} \times \mathrm{U}(1)_{Y}$ remains unbroken and corresponds to the electroweak SM group. However, radiative corrections coming from the fermionic sector of the model, mainly the third quark generation and an additional vector-like quark $T$, give rise to an effective potential that produces further spontaneous symmetry breaking of the $\mathrm{SM} \mathrm{SU}(2)_{\mathrm{L}} \times$ $\mathrm{U}(1)_{Y}$ down to $\mathrm{U}(1)_{\mathrm{em}}$. In this way some of the Goldstone bosons acquire masses quadratic in the cut-off $\Lambda$, which is expected to be of the order of $4 \pi f$ [12-14]. The SM model Higgs boson gets a mass that grows only as the logarithm of $\Lambda$ at the one-loop level. This is the explanation in this setting of why the Higgs boson is expected to be relatively light $\left(115 \mathrm{GeV}<m_{H}<200 \mathrm{GeV}\right)$. The rest of the Goldstone bosons give masses to the different gauge

\footnotetext{
a e-mail: siannah.penaranda@cern.ch
}

bosons through the Higgs mechanism in $\mathrm{SU}(5)$ to $\mathrm{SO}(5)$ or in SM symmetry breaking. Thus, the LH model explains in a natural and elegant way the expected low value of the Higgs boson mass. In addition this model provides a very rich phenomenology, which could be probed in the nextgeneration colliders such as the LHC $[15,16]$. Since the original proposal of the LH, many other little Higgs versions have appeared [17-21]. Some of these models try to improve the consistency and to reduce the need for finetuning in this kind of models [22]. Specially interesting from the phenomenological point of view is the LH version in which the $\mathrm{SU}(5)$ gauged subgroup is just $\mathrm{SU}(2)_{1} \times$ $\mathrm{SU}(2)_{2} \times \mathrm{U}(1)$. In this case, after the first spontaneous broken symmetry, we have only three massive gauge bosons associated to the $\mathrm{SU}(2)_{1-2}$ group, $W^{\prime a}$, and four massless gauge bosons, i.e. the SM gauge bosons [16].

Nevertheless, it is clear that any viable little Higgs model has to fulfill the basic requirement of reproducing the SM model at low energies. This implies, in particular, that one should not only have the proper low energy degrees of freedom, but also one should be able to reproduce the action of the SM model as the low energy effective action of the LH model. In this work we compute the contribution of the $t, b$ and $T$ quarks to the effective potential for the SM Higgs doublet $H=\left(H^{0}, H^{+}\right)$, which gives rise to electroweak symmetry breaking in the LH model. The first terms of this potential are found to have the standard form:

$$
V_{\text {eff }}=-\mu^{2} H H^{\dagger}+\lambda\left(H H^{\dagger}\right)^{2},
$$

with positive $\mu^{2}$ and $\lambda$. Other relevant contributions coming from gauge bosons, scalars and other higher loops are 
expected to go in the opposite direction, but they are also supposed to have a smaller absolute value, so that they do not change the $\mu^{2}$ and $\lambda$ signs. The $\mu^{2}$ sign and value are well known $[11,16]$, and effectively they are the right ones to produce the electroweak symmetry breaking, giving a Higgs mass $m_{H}^{2}=2 \mu^{2}$. However, the full expression for $\lambda$ has not been analyzed in detail. Several relations for the threshold corrections to this parameter in the presence of a $10 \mathrm{TeV}$ cut-off, depending of the UV-completion of the theory, have been reported before [23]. The radiative contributions to $\lambda$, at the one-loop level, have not been computed so far.

The computation of the parameter $\lambda$ is important for several reasons. First, it must be positive for the low energy effective action to make sense (otherwise the theory would not have any vacuum). In addition, from the effective potential above, one gets the simple formula $m_{H}^{2}=2 \lambda v^{2}$, or, equivalently, $\mu^{2}=\lambda v^{2}$, where $v$ is the SM vacuum expectation value $(H=(0, v) / \sqrt{2})$, which is experimentally set to be $v \simeq 245 \mathrm{GeV}$ (for instance by the muon lifetime). By computing the effective action for the Higgs doublet in the context of the LH model and taking into account the $t, b$ and $T$ quarks only, i.e. the modes responsible for the electroweak symmetry breaking, it is possible to obtain $\mu^{2}$ and $\lambda$ in terms of the $\lambda_{T}, f$ and $\Lambda$ parameters of the $\mathrm{LH}$ model, where $\lambda_{T}$ is the Yukawa coupling of $T, f$ is the scale of the $\mathrm{SU}(5) / \mathrm{SO}(5)$ symmetry breaking, and $\Lambda$ is the ultraviolet cut-off (in fact $\lambda$ has also a small dependence on the infrared cut-off $m \sim v$ ). In other words, we find the functions

$$
\begin{aligned}
\mu^{2} & =\mu^{2}\left(\lambda_{T}, f, \Lambda\right)+\ldots, \\
\lambda & =\lambda\left(\lambda_{T}, f, \Lambda\right)+\ldots,
\end{aligned}
$$

where the ellipses include corrections coming from the gauge bosons, scalars and other higher order loops. As is well known, $\mu$ depends on the logarithm of $\Lambda$ at the oneloop level, but $\lambda$ has also a much stronger quadratic dependence on this cut-off. Moreover, according to the previous discussion, the consistency of the low energy theory sets the following highly non-trivial constraint on the LH model parameters:

$$
\mu^{2}\left(\lambda_{T}, f, \Lambda\right)=\lambda\left(\lambda_{T}, f, \Lambda\right) v^{2}+\ldots
$$

In this work we compute the contribution to these functions coming from the $t, b$ and $T$ quarks present in the $\mathrm{LH}$ model at the one-loop level, which are the relevant ones for having symmetry breaking. Then we use this result to illustrate the kind of bounds and restrictions that must be set on the LH model fermion parameters in order to obtain the SM potential from the effective Higgs potential that should also include gauge, scalar and other higher loop contributions [43].

The outline of the paper is as follows: in Sect. 2 we review briefly the $\mathrm{LH}$ model and settle the notation we are going to use. In Sect. 3 we compute the $t, b$ and $T$ quark contribution to the effective action for a constant SM Higgs doublet (i.e. the effective potential) by using standard techniques [24], and we obtain the functions $\mu^{2}=\mu^{2}\left(\lambda_{T}, f, \Lambda\right)$ and $\lambda=\lambda\left(\lambda_{T}, f, \Lambda\right)$. Section 4 is devoted to the study of the above-mentioned constraints, and as an example we use our previous result to show the kind of restrictions that it is possible to set on the LH model parameter space from the complete Higgs effective potential. Finally, in Sect. 5 we summarize our main results and present some conclusions and remarks.

\section{The model}

The LH model is based on the assumption that there is a physical system with a global $G=\mathrm{SU}(5)$ symmetry that is spontaneously broken to a $H=\mathrm{SO}(5)$ symmetry at a high scale $\Lambda$ through a vacuum expectation value of order $f$. Thus the spectrum of the theory will in principle contain 14 Goldstone bosons, including the SM complex doublet $H=\left(H^{0}, H^{+}\right)$. In addition, the $\mathrm{SU}(5)$ subgroup $(\mathrm{SU}(2) \times \mathrm{U}(1))_{1} \times(\mathrm{SU}(2) \times \mathrm{U}(1))_{2}$ is gauged, its diagonal subgroup $(\mathrm{SU}(2) \times \mathrm{U}(1))_{1+2}$ being the $\mathrm{SM}$ electroweak group $\mathrm{SU}(2)_{\mathrm{L}} \times \mathrm{U}(1)_{Y}$. This group remains unbroken after the $\mathrm{SU}(5)$ breaking to $\mathrm{SO}(5)$ and consequently the electroweak gauge bosons $W_{\mu}^{a}$ and $B_{\mu}$ are massless at this level. However, the $(\mathrm{SU}(2) \times \mathrm{U}(1))_{1-2}$ group is spontaneously broken and the corresponding gauge bosons $W_{\mu}^{\prime a}$ and $B_{\mu}^{\prime}$ get masses of order $f$ through the Higgs mechanism. Each of these two gauge groups must commute with a different subgroup $\mathrm{SU}(3)$ that acts non-linearly on the Higgs, i.e. when both weak gauge interactions are included, the Higgs boson is a pseudo-Goldstone boson whose mass is protected by the underlying symmetry, but, conversely, if just one of these interactions is considered, the SU(3) symmetry is recovered [11].

With the global SU(5) symmetry breaking into its subgroup $\mathrm{SO}(5)$, we have 14 Goldstone bosons, which transform under the electroweak group as a real singlet, a real triplet, a complex doublet and a complex triplet. The real singlet and the real triplet become the longitudinal parts of the $B_{\mu}^{\prime}$ and $W_{\mu}^{\prime} a$ bosons through the Higgs mechanism, and the latter two Goldstone boson multiplets can be interpreted as the SM Higgs doublet and an additional complex triplet; i.e., we still have 10 massless Goldstone bosons. These particles will get radiative masses after the introduction of appropriate gauge and Yukawa couplings to the third-generation $t$ and $b$ quarks and an additional vector-like quark $T$, the Yukawa contributions being responsible for the expected sign of the $\mu^{2}$ parameter. Then the magic of the model produces a Higgs mass that is quadratically divergence-free at the one-loop level. Thus we obtain a light Higgs boson in a natural way. The complex triplet is not protected in the same way, and radiative quark corrections make it typically much more massive, thus evading the experimental constraints.

According to the previous discussion, the low energy dynamics of the $\mathrm{LH}$ model can be described by a $(\mathrm{SU}(2) \times$ $\mathrm{U}(1))_{1} \times(\mathrm{SU}(2) \times \mathrm{U}(1))_{2}$ gauged non-linear sigma model based on the coset $K=G / H=\mathrm{SU}(5) / \mathrm{SO}(5)$ [24]. The Goldstone boson fields can be arranged in a $5 \times 5$ matrix $\Sigma$ 
given by

$$
\Sigma=\mathrm{e}^{2 \mathrm{i} \Pi / f} \Sigma_{0}
$$

where

$$
\Sigma_{0}=\left(\begin{array}{lll}
0 & 0 & 1 \\
0 & 1 & 0 \\
1 & 0 & 0
\end{array}\right)
$$

has the proper $\mathrm{SU}(5)$ symmetry breaking structure, $\mathbf{1}$ being the $2 \times 2$ unit matrix, and

$$
\Pi=\left(\begin{array}{ccc}
\xi & \frac{-\mathrm{i}}{\sqrt{2}} H^{\dagger} & \phi^{\dagger} \\
\frac{\mathrm{i}}{\sqrt{2}} H & 0 & \frac{-\mathrm{i}}{\sqrt{2}} H^{*} \\
\phi & \frac{\mathrm{i}}{\sqrt{2}} H^{\mathrm{T}} & \xi^{\mathrm{T}}
\end{array}\right)+\frac{\eta}{\sqrt{20}} \operatorname{diag}(1,1,-4,1,1)
$$

is the Goldstone bosons matrix, with $H=\left(H^{0}, H^{+}\right)$being the SM Higgs doublet, $\eta$ being a real scalar, and $\xi$ and $\phi$ encoding the real triplet and the complex triplet, respectively:

$$
\xi=\left(\begin{array}{cc}
\frac{1}{2} \xi^{0} & \frac{1}{\sqrt{2}} \xi^{+} \\
\frac{1}{\sqrt{2}} \xi^{-} & -\frac{1}{2} \xi^{0}
\end{array}\right)
$$

and

$$
\phi=\left(\begin{array}{cc}
\phi^{0} & \frac{1}{\sqrt{2}} \phi^{+} \\
\frac{1}{\sqrt{2}} \phi^{+} & \frac{1}{\sqrt{2}} \phi^{++}
\end{array}\right) .
$$

The Lagrangian of the gauged non-linear sigma model is given by

$$
\mathcal{L}_{0}=\frac{f^{2}}{8} \operatorname{tr} D_{\mu} \Sigma\left(D^{\mu} \Sigma\right)^{\dagger}
$$

where the covariant derivative is defined by [11]

$$
\begin{aligned}
D_{\mu} \Sigma= & \partial_{\mu} \Sigma-\mathrm{i} \sum_{j=1}^{2} g_{j} W_{j}^{a}\left(Q_{j}^{a} \Sigma+\Sigma Q_{j}^{a \mathrm{~T}}\right) \\
& -\mathrm{i} \sum_{j=1}^{2} g_{j}^{\prime} B_{j}\left(Y_{j} \Sigma+\Sigma Y_{j}^{\mathrm{T}}\right),
\end{aligned}
$$

where $g_{j}$ and $g_{j}^{\prime}$ are the gauge couplings, $Q_{1 i j}^{a}=\sigma_{i j}^{a} / 2$ for $i, j=1,2, Q_{2 i j}^{a}=\sigma_{i j}^{a *} / 2$ for $i, j=4,5$, and zero otherwise, $Y_{1}=\operatorname{diag}(-3,-3,2,2,2) / 10$ and $Y_{2}=\operatorname{diag}(-2,-2,-2$, $3,3) / 10$. By diagonalizing the gauge boson mass matrix contained in this Lagrangian one gets the massless $W$ and $B$ SM bosons and the massive $W^{\prime}$ and $B^{\prime}$ gauge bosons mentioned above as follows:

$$
\begin{aligned}
W^{a} & =c_{\psi} W_{1}^{a}+s_{\psi} W_{2}^{a}, \\
W^{\prime} a & =s_{\psi} W_{1}^{a}-c_{\psi} W_{2}^{a},
\end{aligned}
$$

where

$$
\begin{aligned}
& s_{\psi}=\sin \psi=\frac{g_{1}}{\sqrt{g_{1}^{2}+g_{2}^{2}}}, \\
& c_{\psi}=\cos \psi=\frac{g_{2}}{\sqrt{g_{1}^{2}+g_{2}^{2}}},
\end{aligned}
$$

with $M_{W}=0$ and $M_{W^{\prime}}=f \sqrt{g_{1}^{2}+g_{2}^{2}} / 2$. In a similar way, we have

$$
\begin{aligned}
B & =c_{\psi}^{\prime} B_{1}+s_{\psi}^{\prime} B_{2}, \\
B^{\prime} & =s_{\psi}^{\prime} B_{1}-c_{\psi}^{\prime} B_{2},
\end{aligned}
$$

where

$$
\begin{aligned}
& s_{\psi}^{\prime}=\sin \psi^{\prime}=\frac{g_{1}^{\prime}}{\sqrt{{g^{\prime}}_{1}^{2}+{g^{\prime}}_{2}^{2}}}, \\
& c_{\psi}^{\prime}=\cos \psi^{\prime}=\frac{{g^{\prime}}_{2}}{\sqrt{{g^{\prime}}_{1}^{2}+{g^{\prime}}_{2}^{2}}},
\end{aligned}
$$

where $M_{B}=0$ and $M_{B^{\prime}}=f \sqrt{g_{1}^{\prime 2}+g_{2}^{\prime 2}} / \sqrt{20}$.

A version of the LH models modified such that the gauge subgroup of $\mathrm{SU}(5)$ is $\left[\mathrm{SU}(2) \times \mathrm{SU}(2) \times \mathrm{U}(1)_{Y}\right]$ rather than $\left[\mathrm{SU}(2) \times \mathrm{U}(1)_{Y}\right]^{2}$ has also been introduced [16]. In this case, the covariant derivative is defined by

$$
\begin{aligned}
D_{\mu} \Sigma= & \partial_{\mu} \Sigma-\mathrm{i} \sum_{j=1}^{2} g_{j} W_{j}^{a}\left(Q_{j}^{a} \Sigma+\Sigma Q_{j}^{a \mathrm{~T}}\right) \\
& -\mathrm{i} g^{\prime} B\left(Y \Sigma+\Sigma Y^{\mathrm{T}}\right),
\end{aligned}
$$

where the generators $Q_{j}^{a}$ are the same as in the previous case, and $Y=\frac{1}{2} \operatorname{diag}(-1,-1,0,1,1)$. The field content of the matrix $\Pi$ in $\Sigma$ is the same as in the LH model but now there is no $B^{\prime}$. This model will be considered in Sect. 4 , where some phenomenological consequences of considering the gauge sector are discussed.

Then, at the tree level, the $\mathrm{SU}(2)_{\mathrm{L}} \times \mathrm{U}(1)_{Y} \mathrm{SM}$ gauge group remains unbroken. Spontaneous symmetry breaking of this group is produced radiatively mainly by the quark loops from the third generation, which will be initially denoted by $u$ and $t$; the additional vector-like quark will be denoted by $U$. The interactions between these fermions and the Goldstone bosons are given by the Yukawa Lagrangian:

$$
L_{\mathrm{Yuk}}=-\frac{\lambda_{1}}{2} f \bar{u}_{\mathrm{R}} \epsilon_{m n} \epsilon_{i j k} \Sigma_{i m} \Sigma_{j n} \chi_{\mathrm{L} k}-\lambda_{2} f \bar{U}_{\mathrm{R}} U_{\mathrm{L}}+\text { h.c. }
$$

where $m, n=4,5, i, j=1,2,3$, and

$$
\begin{gathered}
\bar{u}_{\mathrm{R}}=c \bar{t}_{\mathrm{R}}+s \bar{T}_{\mathrm{R}}, \\
\bar{U}_{\mathrm{R}}=-s \bar{t}_{\mathrm{R}}+c \bar{T}_{\mathrm{R}},
\end{gathered}
$$

with

$$
\begin{aligned}
& c=\cos \theta=\frac{\lambda_{2}}{\sqrt{\lambda_{1}^{2}+\lambda_{2}^{2}}}, \\
& s=\sin \theta=\frac{\lambda_{1}}{\sqrt{\lambda_{1}^{2}+\lambda_{2}^{2}}},
\end{aligned}
$$

and

$$
\chi_{\mathrm{L}}=\left(\begin{array}{c}
u \\
b \\
U
\end{array}\right)_{\mathrm{L}}=\left(\begin{array}{c}
t \\
b \\
T
\end{array}\right)_{\mathrm{L}}
$$


Here $t, b$ and $T$ are the mass eigenvectors coming from the mass matrix included in the Yukawa Lagrangian with eigenvalues $m_{t}=m_{b}=0$ and $m_{T}=f \sqrt{\lambda_{1}^{2}+\lambda_{2}^{2}}$. Thus the quark $t$ is massless, and it acquires mass only when the electroweak symmetry is broken, contrary to the quark $T$, which is massive already at this level.

Next, the Yukawa Lagrangian can be written

$$
L_{\mathrm{Yuk}}=\bar{\chi}_{\mathrm{R}} \hat{I}_{3 \times 3} \chi_{\mathrm{L}}+\text { h.c. },
$$

$\hat{I}_{3 \times 3}$ being the interaction matrix defined below, and

$$
\chi_{\mathrm{R}}=\left(\begin{array}{c}
t \\
b \\
T
\end{array}\right)_{\mathrm{R}}
$$

Since we are interested in the computation of the fermion contribution to the SM Higgs effective potential, we set $\xi=$ $\phi=\eta=0$, and thus the interaction matrix $\hat{I}$ is given by

$$
\hat{I}=\left(\begin{array}{ccc}
-\sqrt{2} \lambda_{1} c H^{0} \Theta & -\sqrt{2} \lambda_{1} c H^{+} \Theta & \lambda_{1} c \frac{H H^{\dagger}}{f} \Theta^{\prime} \\
0 & 0 & 0 \\
-\sqrt{2} \lambda_{1} s H^{0} \Theta & -\sqrt{2} \lambda_{1} s H^{+} \Theta & \lambda_{1} s \frac{H H^{\dagger}}{f} \Theta^{\prime}
\end{array}\right),
$$

where $\Theta$ and $\Theta^{\prime}$ are functions of $H H^{\dagger} / f^{2}$ whose expansion starts as follows:

$$
\begin{aligned}
& \Theta\left(\frac{H H^{\dagger}}{f^{2}}\right)=1-\frac{2 H H^{\dagger}}{3 f^{2}}+\ldots \\
& \Theta^{\prime}\left(\frac{H H^{\dagger}}{f^{2}}\right)=1-\frac{H H^{\dagger}}{3 f^{2}}+\ldots
\end{aligned}
$$

Therefore the complete Lagrangian for the quarks is

$$
L_{\chi}=L_{0}+L_{\mathrm{Yuk}}=\bar{\chi}_{\mathrm{R}}(\mathrm{i} \not \partial-M+\hat{I}) \chi_{\mathrm{L}}+\text { h.c. },
$$

with $M=\operatorname{diag}\left(0,0, m_{T}\right)$.

In the LH model electroweak symmetry breaking is produced mainly by the three quarks included in the above Lagrangian, whilst the gauge bosons and the complex triplet tend to restore the symmetry. In the following we will consider only the effect of the quarks on the effective Higgs potential.

\section{The effective Higgs action and potential}

In order to compute the leading fermion contribution to the effective Higgs potential we will start from the effective Higgs action obtained from the $t, b$ and $T$ quarks at the one-loop level, which is an exact computation in this case since the action is quadratic in these fields. Thus, this effective action is given by

$$
\mathrm{e}^{\mathrm{i} S_{\text {eff }}[H]}=\int[\mathrm{d} \chi][\mathrm{d} \bar{\chi}] \mathrm{e}^{\mathrm{i} S[H, \chi, \bar{\chi}]},
$$

with

$$
S[H, \chi, \bar{\chi}]=\int \mathrm{d}^{4} x\left(\partial_{\mu} H \partial^{\mu} H^{\dagger}+L_{\chi}\right) .
$$

By using standard techniques [24], we obtain the following result for the effective action:

$$
S_{\text {eff }}[H]=\int \mathrm{d}^{4} x \partial_{\mu} H \partial^{\mu} H^{\dagger}+\Delta S,
$$

with

$$
\Delta S=-\mathrm{i} \operatorname{Tr} \log (\mathrm{i} \not \partial-M+\hat{I})=-\mathrm{i} \operatorname{Tr}(1+G \hat{I}),
$$

where we have neglected a constant; it is irrelevant for the computation of the effective action. The operator $G=$ $(\mathrm{i} \not \partial-M)^{-1}$ is just the propagator for the free quarks, which is given by

$$
G^{a b}(x, y) \equiv \int \mathrm{d} \widetilde{k} \mathrm{e}^{-\mathrm{i} p(x-y)}(\not p-M)_{a b}^{-1} .
$$

Here $\mathrm{d} \widetilde{k} \equiv \mathrm{d}^{4} k /(2 \pi)^{4}$. By expanding the logarithm, the effective action can be written as

$$
\Delta S=-\mathrm{i} \operatorname{Tr} \Sigma_{k=1}^{\infty} \frac{(-1)^{k+1}}{k}(G \hat{I})^{k}=\Sigma_{k=1}^{\infty} \Delta S^{(k)} .
$$

Now in order to obtain the effective potential we only have to consider constant Higgs fields, i.e. we set $\partial_{\mu} H=0$. Thus we have

$$
\left.\Delta S\right|_{H=\text { const. }}=-\int \mathrm{d}^{4} x V_{\text {eff }}(H) .
$$

In the following we will take $H$ as a constant. The effective potential can be computed as a power series in $H H^{\dagger} / f^{2}$, with arbitrarily higher powers of this parameter. However, in order to produce electroweak symmetry breaking, it is sufficient to compute just the first two terms of this expansion. Thus the effective potential can be written as in (1).

It is then not difficult to see that the computation of the $\mu^{2}$ and $\lambda$ parameters requires $\Delta S$ to be considered up to the fourth term. The generic one-loop diagrams that must be computed are shown in Fig. 1. By using well known methods it is straightforward to obtain the different contributions after some work. The first one $(k=1)$ corresponds to the first two diagrams in Fig. 1, and it is given by

$$
\begin{aligned}
\Delta S^{(1)}[H] & =-\mathrm{i} \operatorname{Tr}(G \hat{I}) \\
& =-\frac{4}{3} \lambda_{1} m_{T} f s \int \mathrm{d}^{4} x\left(\frac{H H^{\dagger}}{f^{2}}-\frac{\left(H H^{\dagger}\right)^{2}}{f^{4}}\right) \\
& \times I_{0}\left(m_{T}^{2}\right),
\end{aligned}
$$

where the divergent integral $I_{0}\left(m_{T}^{2}\right)$ is

$$
I_{0}\left(m_{T}^{2}\right)=\int \mathrm{d} \tilde{p} \frac{\mathrm{i}}{p^{2}-m_{T}^{2}} .
$$

In order to make sense of this integral we will use an ultraviolet cut-off $\Lambda$, where our effective description of the low energy dynamics breaks down. The result is

$$
I_{0}\left(m_{T}^{2}\right)=\frac{1}{(4 \pi)^{2}}\left[\Lambda^{2}-m_{T}^{2} \log \left(\frac{\Lambda^{2}}{m_{T}^{2}}+1\right)\right]
$$



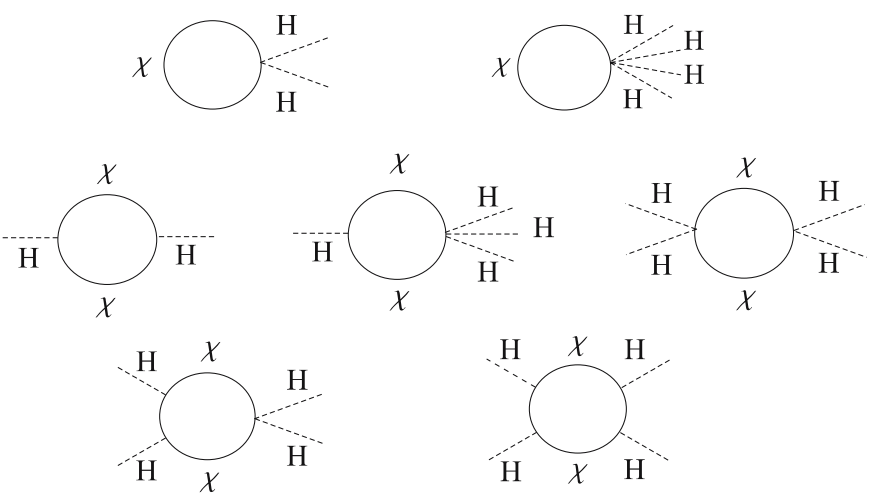

Fig. 1. One-loop diagrams. $H=\left(H^{0}, H^{+}\right)$and $\chi \equiv t, b, T$, with all possible combinations of these particles in the loop diagrams

For $k=2$ (see the three generic diagrams on the second line of Fig. 1), one gets

$$
\begin{aligned}
& \Delta S^{(2)}[H] \\
& \quad=\frac{i}{2} \operatorname{Tr}(G \hat{I})^{2}=4 \lambda_{1}^{2} \int \mathrm{d}^{4} x H H^{\dagger}\left(c^{2} I_{0}(0)+s^{2} I_{0}\left(m_{T}^{2}\right)\right) \\
& \quad+2 \lambda_{1}^{2} \int \mathrm{d}^{4} x \frac{\left(H H^{\dagger}\right)^{2}}{f^{4}}\left(I_{0}(0)+2 m_{T}^{2} s^{2} I_{1}\left(m_{T}^{2}\right)\right) \\
& \quad-\frac{16}{3} \lambda_{1}^{2} \int \mathrm{d}^{4} x \frac{\left(H H^{\dagger}\right)^{2}}{f^{4}}\left(c^{2} I_{0}(0)+s^{2} I_{0}\left(m_{T}^{2}\right)\right)
\end{aligned}
$$

where the new divergent integral $I_{1}\left(m_{T}^{2}\right)$ properly regularized is given by

$$
\begin{aligned}
I_{1}\left(m_{T}^{2}\right)= & \int \mathrm{d} \tilde{p} \frac{\mathrm{i}}{\left(p^{2}-m_{T}^{2}\right)^{2}}=-\frac{1}{(4 \pi)^{2}} \\
& \times\left[\log \left(\frac{\Lambda^{2}}{m_{T}^{2}}+1\right)-\frac{1}{1+\frac{m_{T}^{2}}{\Lambda^{2}}}\right] .
\end{aligned}
$$

The $k=3$ contribution (see the first diagram in the bottom line of Fig. 1) is

$$
\begin{aligned}
\Delta S^{(3)}[H]= & -\frac{\mathrm{i}}{3} \operatorname{Tr}(G \hat{I})^{3} \\
= & -8 m_{T} \lambda_{1}^{3} s \int \mathrm{d}^{4} x \frac{\left(H H^{\dagger}\right)^{2}}{f} \\
& \times\left(c^{2} I_{2}\left(m_{T}^{2}\right)+s^{2} I_{1}\left(m_{T}^{2}\right)\right),
\end{aligned}
$$

where the divergent integral $I_{2}\left(m_{T}^{2}\right)$ can be written

$$
I_{2}\left(m_{T}^{2}\right)=\int \mathrm{d} \tilde{p} \frac{\mathrm{i}}{p^{2}\left(p^{2}-m_{T}^{2}\right)}=-\frac{1}{(4 \pi)^{2}} \log \left(\frac{\Lambda^{2}}{m_{T}^{2}}+1\right) .
$$

Finally, for $k=4$ we get

$$
\begin{aligned}
\Delta S^{(4)}[H]= & \frac{\mathrm{i}}{4} \operatorname{Tr}(G \hat{I})^{4} \\
= & 4 \lambda_{1}^{4} \int \mathrm{d}^{4} x\left(H H^{\dagger}\right)^{2}\left(s^{4} I_{1}\left(m_{T}^{2}\right)+2 s^{2} c^{2} I_{2}\left(m_{T}^{2}\right)\right. \\
& \left.+c^{4} I_{2}(0)\right) .
\end{aligned}
$$

Here we need to compute $I_{2}(0)$. This integral is not only ultraviolet-divergent but also infrared-divergent. Thus, we need to introduce a new infrared cut-off $m$ (obviously the natural value for this cut-off is of the order of $v$, i.e. the scale of electroweak symmetry breaking). Then we find

$$
I_{2}(0)=\int \mathrm{d} \tilde{p} \frac{\mathrm{i}}{p^{4}}=-\frac{1}{(4 \pi)^{2}} \log \left(\frac{\Lambda^{2}}{m^{2}}\right) .
$$

Therefore, by using the previous results, it is possible to write the effective Higgs potential parameters as ${ }^{1}$

$$
\mu^{2}=N_{c} \frac{m_{T}^{2} \lambda_{t}^{2}}{4 \pi^{2}} \log \left(1+\frac{\Lambda^{2}}{m_{T}^{2}}\right)
$$

and

$$
\begin{aligned}
\lambda= & \frac{N_{c}}{(4 \pi)^{2}}\left[2\left(\lambda_{t}^{2}+\lambda_{T}^{2}\right) \frac{\Lambda^{2}}{f^{2}}-\log \left(1+\frac{\Lambda^{2}}{m_{T}^{2}}\right)\right. \\
& \times\left(-\frac{2 m_{T}^{2}}{f^{2}}\left(\frac{5}{3} \lambda_{t}^{2}+\lambda_{T}^{2}\right)+4 \lambda_{t}^{4}+4\left(\lambda_{T}^{2}+\lambda_{t}^{2}\right)^{2}\right) \\
& \left.-4 \lambda_{T}^{2} \frac{1}{1+\frac{m_{T}^{2}}{\Lambda^{2}}}\left(\frac{m_{T}^{2}}{f^{2}}-2 \lambda_{t}^{2}-\lambda_{T}^{2}\right)-4 \lambda_{t}^{4} \log \left(\frac{\Lambda^{2}}{m^{2}}\right)\right],
\end{aligned}
$$

where $N_{c}$ is the number of colors and $\lambda_{t}$ and $\lambda_{T}$ are, respectively, the SM top Yukawa coupling and the heavy top Yukawa coupling, given by ${ }^{2}$

$$
\begin{aligned}
\lambda_{t} & =\frac{\lambda_{1} \lambda_{2}}{\sqrt{\lambda_{1}^{2}+\lambda_{2}^{2}}} \\
\lambda_{T} & =\frac{\lambda_{1}^{2}}{\sqrt{\lambda_{1}^{2}+\lambda_{2}^{2}}} .
\end{aligned}
$$

There are several comments on this result worthwhile stressing. First, the effective potential depends on $H$ through the combination $|H|^{2}=H H^{\dagger}$, thus reflecting the fact that the radiative corrections considered preserve the $\mathrm{SM} \mathrm{SU}(2)_{\mathrm{L}} \times \mathrm{U}(1)_{Y}$ symmetry. However, $\mu^{2}>0$ and $\lambda>0$, which are the right signs for these corrections to spontaneously break this symmetry down to $\mathrm{U}(1)_{\mathrm{em}}$. Thus the minima of the effective potential occur whenever $|H|^{2}=v^{2} / 2 \equiv \mu^{2} /(2 \lambda)$. By choosing the new vacuum as the state $H=(0, v) / \sqrt{2}$, we recover the above-mentioned SM symmetry breaking. In particular we find that the physical Higgs boson mass is given by $m_{H}^{2}=2 \lambda v^{2}$. Notice that for $\lambda<0$ the model would be inconsistent, and that for $\mu^{2}<0$ there would not be spontaneous symmetry breaking.

In spite of having found apparently the same results as in the SM, the LH model has, however, a number of nice properties. First of all, as we have shown, the Higgs potential parameters can be computed in terms of other, more fundamental, parameters. Therefore there is no need to introduce the parameters ad hoc in order to get the

${ }^{1}$ Our results agree with previous ones for $\mu^{2}$ (see, for example, [2] and references therein).

2 Here we assume that $m_{t}=\lambda_{t} v$. 
appropriate symmetry breaking as it happens in the SM. Symmetry breaking appears as a result of the dynamics, through third-generation quarks radiative corrections, and not as a tree-level consequence of the SM Lagrangian. On the other hand, the Higgs mass can be written as $m_{H}^{2}=2 \mu^{2}$ and $\mu^{2}$ is only a logarithmic-divergent quantity at the oneloop level. Therefore, the Higgs mass is not only light, as precision tests of the SM seems to suggest, but also it is free from the undesired quadratic divergences that appear in the original formulation of the SM at one-loop level. Notice also that the quadratic divergences appearing in $\lambda$ do not alter this result.

Once spontaneous breaking of the SM symmetry has taken place, the Yukawa Lagrangian given above gives rise to a new mass matrix for the $t$ and $T$ quarks (the $b$ quark remains massless). This mass matrix can be diagonalized through a rotation of the left chiral states $t_{\mathrm{L}}$ and $T_{\mathrm{L}}$ given by the angle $\theta_{\mathrm{L}}$, which, for $v \ll f$, can be written

$$
\sin \theta_{\mathrm{L}} \simeq\left(\frac{\lambda_{1}^{2}}{\lambda_{1}^{2}+\lambda_{2}^{2}}\right) \frac{v}{f}
$$

and by another rotation of the right states $t_{\mathrm{R}}$ and $T_{\mathrm{R}}$ given by the angle $\theta_{\mathrm{R}}$,

$$
\sin \theta_{\mathrm{R}} \simeq s\left[1-c^{2}\left(\frac{1}{2}-\left(\frac{\lambda_{1}^{2}}{\lambda_{1}^{2}+\lambda_{2}^{2}}\right)\right) \frac{v^{2}}{f^{2}}\right] .
$$

After these rotations the new mass eigenvalues become

$$
m_{t}=\lambda_{t} v\left[1+\left(-\frac{1}{3}+\frac{1}{2} \frac{\lambda_{1}^{2}}{\lambda_{1}^{2}+\lambda_{2}^{2}}\left(1-\frac{\lambda_{1}^{2}}{\lambda_{1}^{2}+\lambda_{2}^{2}}\right)\right) \frac{v^{2}}{f^{2}}\right]
$$

and

$$
m_{T}=\frac{\lambda_{t}^{2}+\lambda_{T}^{2}}{\lambda_{T}} f-\frac{1}{2} \frac{\lambda_{1}^{2}}{\sqrt{\lambda_{1}^{2}+\lambda_{2}^{2}}}\left(1-\frac{\lambda_{1}^{2}}{\lambda_{1}^{2}+\lambda_{2}^{2}}\right) \frac{v^{2}}{f} .
$$

\section{Constraints on the $\mathrm{LH}$ parameter space}

Whatever model one considers as a candidate for physics beyond the SM, consistency with the present experimental data is a key requirement. It is well known that indirect constraints from precision electroweak measurements on new physics at the TeV scale are severe. There exist several studies of the corrections to electroweak precision observables in little Higgs models, exploring whether there are regions of the parameter space in which the model is consistent with the data $[1,2,15,25-40]$.

The effective Higgs potential parameters are related with the vacuum expectation value $v$ through $\mu^{2}=\lambda v^{2}$. By imposing this condition, we could extract crucial information on the allowed region of the parameter space in the $\mathrm{LH}$ model. To show this, we will consider here the case of the heavy quark contribution to the Higgs potential. In spite of the fact that other contributions, coming from the gauge bosons, scalars and other higher loops, are relevant, the fermionic sector provides a good illustration of the kind of constraints that it will be possible to set on the LH parameter space by means of the complete effective Higgs potential.

The contributions from the fermion sector of the model to $\mu^{2}$ and $\lambda$ are summarized in (39) and (40), respectively. The relevant free parameters of the model are the heavy top mass $m_{T}$, the coupling constant $\lambda_{T}$, the symmetry breaking scale $f$, and the scale $\Lambda$. However, there are several relations between them which are worth remarking on. First of all, at lowest order we have

$$
m_{T}=f \frac{\lambda_{t}^{2}+\lambda_{T}^{2}}{\lambda_{T}}
$$

which in principle, could be tested by LHC experiments $[2$, 16]. This relation is crucial for the cancelation of quadratic divergence contributions to the Higgs boson mass. Besides, considering $m_{T}$ to be much larger than $2 \mathrm{TeV}$ would imply a large amount of fine-tuning in the Higgs potential, and thus the heavy top should be below about $2 \mathrm{TeV}\left(m_{T} \lesssim 2.5 \mathrm{TeV}\right)[11,16]$. From the top mass it is also possible to set bounds on the couplings: $\lambda_{1}, \lambda_{2} \geq m_{t} / v$ or $\lambda_{1} \lambda_{2} \geq 2\left(m_{t} / v\right)^{2}$ [15]. As a consequence, we get the bound $\lambda_{T} \gtrsim 0.5$, which has been considered in our analysis. For the purpose of illustration the dependence of $m_{T}$ on $\lambda_{T}$ and $f$ is shown in Fig. 2a for $0.5<\lambda_{T}<2.5$ and $0.8 \mathrm{TeV}<f<2 \mathrm{TeV}$. Clearly $m_{T}$ grows linearly with $f$. Then the condition $m_{T} \lesssim 2 \mathrm{TeV}$ implies $f \lesssim 1 \mathrm{TeV}$. For $\lambda_{T}>1.7$ we get values of the heavy top mass above $2 \mathrm{TeV}$ for $f=1 \mathrm{TeV}$ (Fig. $2 \mathrm{~b}$ ). We note that once spontaneous symmetry breaking of the SM is produced, the heavy top mass is reduced by $\mathcal{O}\left(v^{2} / f^{2}\right)$ terms (see (45)), but the reduction is only about $0.01 \mathrm{TeV}$. Therefore the previous discussion does not change in a significant way. On the other hand, $\Lambda$ is expected to be of the order of $\Lambda \sim 4 \pi f[12-14]$ and, in addition, electroweak precision tests seem to indicate an experimental lower bound of $\Lambda \gtrsim 10 \mathrm{TeV}[41,42]$.

Taking into account the above restrictions on the three parameters $\lambda_{T}, f$ and $\Lambda$, we now illustrate the kind of constraints that could be set on the LH parameters from the condition $\mu^{2}=\lambda v^{2}$. For the numerical analysis we consider the following ranges: $0.5<\lambda_{T}<2,10 \mathrm{TeV}<\Lambda<12 \mathrm{TeV}$ and, accordingly, $0.8 \mathrm{TeV}<f<1 \mathrm{TeV}$. Let us first describe the behavior of $\mu^{2}$ versus $\lambda_{T}, f$ and $\Lambda$. In general $\mu^{2}$ corrections increase with $f$ for $0.5<\lambda_{T}<2$, having a minimum for a certain value of $\lambda_{T}$, which corresponds to a minimum of $m_{T}$. These corrections also increase with $\Lambda$, but less dramatically. We find that the lowest value of $\mu$ is $\mu=0.48 \mathrm{TeV}$, corresponding to $\lambda_{T}=0.72, f=0.8 \mathrm{TeV}$ and $\Lambda=10 \mathrm{TeV}$.

Once the corrections to the quartic coupling $\lambda$ have been computed (see (40)), the consistency of the LH model is constrained by the non-trivial condition (3). The leading contributions of $t, b$ and $T$ to $\lambda$ in (40) had not been given so far. In the following our purpose will be to use them to illustrate the kind of constraints on the LH model that can be set from the relation between $\lambda$ and $\mu^{2}$. 


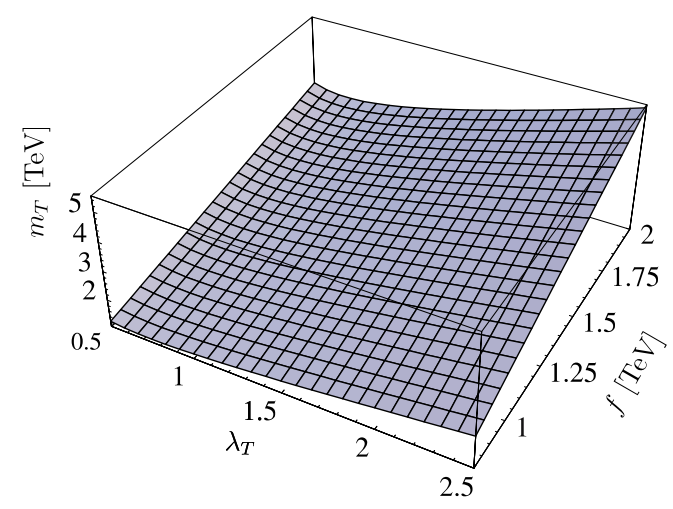

(a)

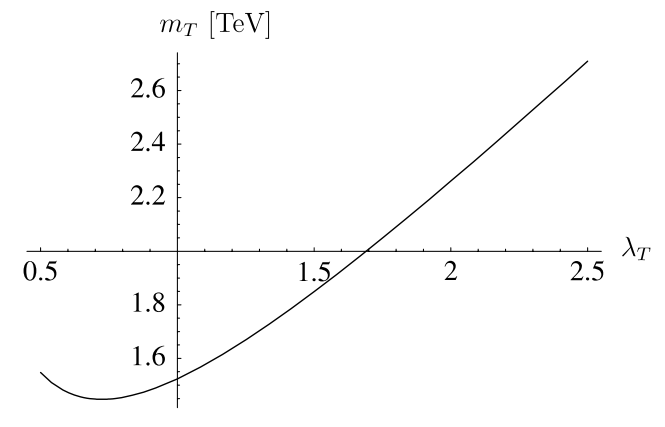

(b)
Fig. 2. (a) $m_{T}$ as a function of $\lambda_{T}$ and $f$, with $0.5<\lambda_{T}<$ 2.5 and $0.5<f<2$ and (b) $m_{T}$ as a function of $\lambda_{T}$ for $f=1 \mathrm{TeV}$
In general, the corrections to $\lambda$ grow with the scale $\Lambda$, but, conversely, we also find that they decrease with the symmetry breaking scale $f$. Figure 3 shows the surface of solutions for this non-trivial condition. Clearly the allowed region of the parameter space is considerably reduced. The lowest $\mu$ value satisfying (3) is $\mu=0.52 \mathrm{TeV}$, when $f=0.85 \mathrm{TeV}, \lambda_{T}=0.52$ and $\Lambda=10 \mathrm{TeV}$.

However, it is well known that $\mu$ is forced by the data to be at most of the order of $200 \mathrm{GeV}$. Therefore, other contributions must be included in order to obtain the expected $\mu$ value. Notice that the Higgs mass parameter in the LH model also receives contributions (not included here) from the vector boson sector (one-loop correction) and from the scalar sector (two-loop correction), which are opposite in sign to the fermion contribution computed here.

Concerning the gauge bosons interactions, we may consider the two different models described in Sect. 2: the original LH with two U(1) groups (model I) and the other one with just one $\mathrm{U}(1)$ group (model $I I$ ). The contributions from the vector bosons to the mass term of the Higgs field in each case are given, respectively, at one-loop level,

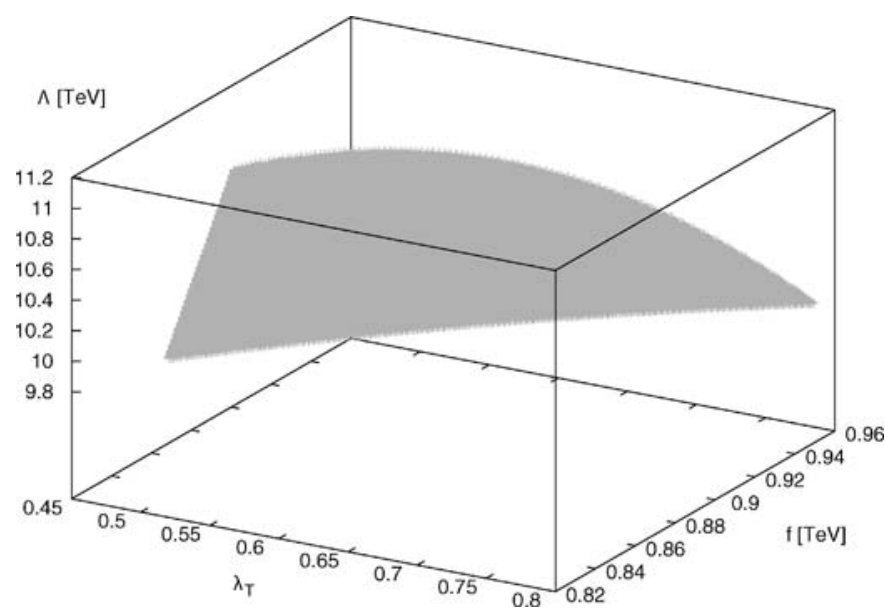

Fig. 3. Values of $\Lambda, f$ and $\lambda_{T}$, with $0.5<\lambda_{T}<2,0.8 \mathrm{TeV}<f<$ $1 \mathrm{TeV}$ and $10 \mathrm{TeV}<\Lambda<12 \mathrm{TeV}$, that satisfy the condition $\mu^{2}=$ $\lambda v^{2}$ by

$$
\begin{aligned}
\mu_{\mathrm{g}}^{2 \mathrm{I}}= & -\frac{3}{64 \pi^{2}}\left(3 g^{2} M_{W^{\prime}}^{2} \log \left(\frac{\Lambda^{2}}{M_{W^{\prime}}^{2}}+1\right)\right. \\
& \left.+g^{\prime 2} M_{B^{\prime}}^{2} \log \left(\frac{\Lambda^{2}}{M_{B^{\prime}}}+1\right)\right), \\
\mu_{\mathrm{g}}^{2 \mathrm{II}}= & -\frac{3}{64 \pi}\left(3 g^{2} M_{W^{\prime}}^{2} \log \left(\frac{\Lambda^{2}}{M_{W^{\prime}}^{2}}+1\right)+g^{\prime 2} \Lambda^{2}\right),
\end{aligned}
$$

where $M_{W^{\prime}}$ and $M_{B^{\prime}}$ are the heavy gauge boson masses,

$$
M_{W^{\prime}}=\frac{f g}{2 \cos \psi \sin \psi} \text { and } M_{B^{\prime}}=\frac{f g^{\prime}}{\sqrt{20} \cos \psi^{\prime} \sin \psi^{\prime}},
$$

with $\psi$ and $\psi^{\prime}$ being the mixing angles for the $W^{\prime}$ and $B^{\prime}$ states. Note that the different results for these two models come from the fact that there is no $B^{\prime}$ in model II.

Let us now study how these vector boson contributions could help to keep $\mu$ of order $200 \mathrm{GeV}$. In order to avoid the gauge masses being too small or too big, we impose $0.1<$ $\cos \psi<0.9$ and $0.1<\cos \psi^{\prime}<0.4$. For model $I$ we find that the minimum value for $\mu$ equals $0.338 \mathrm{TeV}$ for $\lambda_{T} \simeq 0.7$, $f \simeq 0.8 \mathrm{TeV}, \Lambda \simeq 10 \mathrm{TeV}, \cos \psi \simeq 0.1$ and $\cos \psi^{\prime} \simeq 0.1$. In the case of model II we obtain a minimum for $\mu$ of $0.2 \mathrm{TeV}$ at $\lambda_{T} \simeq 0.65, f \simeq 0.8 \mathrm{TeV}, \Lambda \simeq 11.9 \mathrm{TeV}$, and $\cos \psi \simeq 0.1$. Therefore, we find that in model $I I$ it is more natural to find a light Higgs boson than in model I. Note, however, that two-loop scalar corrections may also play an important role.

\section{Conclusions}

In this work we have computed and analyzed the fermion contributions to the low energy effective Higgs potential, and we have illustrated the kind of constraints on the possible values of the LH parameters that can be set by requiring the complete LH effective Higgs potential to reproduce exactly the SM potential. 
We have explored the region of the $\lambda_{T}, f$ and $\Lambda$ parameter space compatible with the condition $\mu^{2}=\lambda v^{2}$, taking $m_{T} \leq 2 \mathrm{TeV}$ and $\Lambda \sim 4 \pi f$. For this purpose we have computed the leading fermion contributions to the quartic coupling $\lambda$ at the one-loop level. Since the values obtained for $\mu$ are relatively high, the inclusion of the gauge and the scalar sector of the model is needed to reduce $\mu$ to its expected value. Therefore, more detailed computations, including the full one-loop gauge boson and the relevant twoloop Goldstone boson contribution, are needed in order to establish the validity of the LH model and its compatibility with the present phenomenological constraint, including the precise form of the Higgs potential. Work is in progress in this direction [43].

Acknowledgements. This work is supported by DGICYT (Spain) under project number BPA2005-02327. The work of S.P. has been partially supported by the European Union under contract No. MEIF-CT-2003-500030. L.T. would like to thank Javier Almeida Linares (UCM, Spain) and Javier Rodriguez Laguna (SISSA, Italy) for their valuable guidance to $\mathrm{C}++$ programming.

\section{References}

1. M. Schmaltz, D. Tucker-Smith, Ann. Rev. Nucl. Part. Sci. 55, 229 (2005) [hep-ph/0502182]

2. M. Perelstein, Prog. Part. Nucl. Phys. 58, 247 (2007) [hep$\mathrm{ph} / 0512128]$

3. H. Georgi, A. Pais, Phys. Rev. D 10, 539 (1974)

4. H. Georgi, A. Pais, Phys. Rev. D 12, 508 (1975)

5. M.J. Dugan, H. Georgi, D.B. Kaplan, Nucl. Phys. B 254, 299 (1985)

6. H. Georgi, D.B. Kaplan, Phys. Lett. B 145, 216 (1984)

7. H. Georgi, D.B. Kaplan, Phys. Lett. B 136, 183 (1984)

8. H. Georgi, D.B. Kaplan, P. Galison, Phys. Lett. B 143, 152 (1984)

9. D.B. Kaplan, H. Georgi, S. Dimopoulos, Phys. Lett. B 136, 187 (1984)

10. S. Dimopoulos, J. Preskill, Nucl. Phys. B 199, 206 (1982)

11. N. Arkani-Hamed, A.G. Cohen, E. Katz, A.E. Nelson, JHEP 0207, 034 (2002) [hep-ph/0206021]

12. A. Manohar, H. Georgi, Nucl. Phys. B 234, 189 (1984)

13. M.A. Luty, Phys. Rev. D 57, 1531 (1998) [hep-ph/9706235]

14. A.G. Cohen, D.B. Kaplan, A.E. Nelson, Phys. Lett. B 412, 301 (1997) [hep-ph/9706275]

15. T. Han, H.E. Logan, B. McElrath, L.T. Wang, Phys. Rev. D 67, 095004 (2003) [hep-ph/0301040]

16. M. Perelstein, M. Peskin, A. Pierce, Phys. Rev. D 69, 075002 (2004) [hep-ph/0310039]
17. I. Low, W. Skiba, D. Smith, Phys. Rev. D 66, 072001 (2002) [hep-ph/0207243]

18. D.E. Kaplan, M. Schmaltz, JHEP 0310, 039 (2003) [hep$\mathrm{ph} / 0302049]$

19. S. Chang, J.G. Wacker, Phys. Rev. D 69, 035002 (2004) [hep-ph/0303001]

20. W. Skiba, J. Terning, Phys. Rev. 68, 075001 (2003) [hep$\mathrm{ph} / 0305302]$

21. S. Chang, JHEP 0312, 057 (2003) [hep-ph/0306034]

22. J.A. Casas, J.R. Espinosa, I. Hidalgo, JHEP 0503, 038 (2005) [hep-ph/0502066]

23. F. Bazzocchi, M. Fabbrichesi, M. Piai, Phys. Rev. D 72, 095019 (2005) [hep-ph/0506175]

24. A. Dobado, A. Gómez-Nicola, A.L. Maroto, J.R. Peláez, Effective Lagrangians for the Standard Model (Springer, Heidelberg, 1997)

25. Z. Han, W. Skiba, Phys. Rev. D 72, 035005 (2005) [hep$\mathrm{ph} / 0506206]$

26. H.E. Logan, Phys. Rev. D 70, 115003 (2004) [hep-ph/ 0405072]

27. T. Han, H.E. Logan, B. McElrath, L.T. Wang, Phys. Lett. B 563, 191 (2003)

28. T. Han, H.E. Logan, B. McElrath, L.T. Wang, Phys. Lett. B B603, 257 (2004) [hep-ph/0302188] [Erratum]

29. C. Csaki, J. Hubisz, G.D. Kribs, P. Meade, J. Terning, Phys. Rev. D 67, 115002 (2003) [hep-ph/0211124]

30. C. Csaki, J. Hubisz, G.D. Kribs, P. Meade, J. Terning, Phys. Rev. D 68, 035009 (2003) [hep-ph/0303236]

31. J.L. Hewett, F.J. Petriello, T.G. Rizzo, JHEP 0310, 062 (2003) [hep-ph/0211218]

32. T. Gregoire, D.R. Smith, J.G. Wacker, Phys. Rev. D 69, 115008 (2004) [hep-ph/0305275]

33. M.C. Chen, S. Dawson, Phys. Rev. D 70, 015003 (2004) [hep-ph/0311032]

34. W. Kilian, J. Reuter, Phys. Rev. D 70, 015004 (2004) [hep$\mathrm{ph} / 0311095]$

35. G. Marandella, C. Schappacher, A. Strumia, Phys. Rev. D 72, 035014 (2005) [hep-ph/0502096]

36. M.C. Chen, Mod. Phys. Lett. A 21, 621 (2006) [hep$\mathrm{ph} / 0601126]$

37. S.R. Choudhury, A.S. Cornell, N. Gaur, A. Goyal, hep$\mathrm{ph} / 0604162$

38. J.A. Conley, J. Hewett, M.P. Le, Phys. Rev. D 72, 115014 (2005) [hep-ph/0507198]

39. C.O. Dib, R. Rosenfeld, A. Zerwekh, AIP Conf. Proc. 815, 296 (2006) [hep-ph/0509013]

40. Z. Berezhiani, P.H. Chankowski, A. Falkowski, S. Pokorski, Phys. Rev. Lett. 96, 031801 (2006) [hep-ph/0509311]

41. R. Barbieri, A. Strumia, hep-ph/0007265

42. R. Barbieri, A. Pomarol, R. Rattazzi, A. Strumia, Nucl. Phys. B 703, 127 (2004) [hep-ph/0405040]

43. A. Dobado, L. Tabares, S. Peñaranda, hep-ph/0612131 